\title{
The effect of maternal poliovirus antibodies on the immune responses of infants to poliovirus vaccines
}

\author{
Siyue Jia ${ }^{1 \dagger}$, Rong Tang ${ }^{1 \dagger}$, Guifan $\mathrm{Li}^{2}$, Yuemei Hu${ }^{1}$ and Qi Liang ${ }^{1 *}$
}

\begin{abstract}
Background: Maternal poliovirus antibodies could provide passive immunity to the newborns from poliovirus infection during their first few months of life, but they may impair the immune responses of infants to the poliovirus vaccine as well. In our study, we pooled the data from three clinical trials of the inactivated poliovirus vaccine (IPV) based on Sabin strains to investigate the effect of maternal poliovirus antibodies on the immune responses of infants to poliovirus vaccines.

Methods: There were five groups in the pooled analysis, including low-dose Sabin IPV, medium-dose Sabin IPV, high-dose Sabin IPV, control Sabin IPV, and control Salk IPV groups. We reclassified the infants in different groups according to their maternal poliovirus antibodies by two methods, the first one included maternal antibody negative $(<1: 8)$ and maternal antibody positive $(\geq 1: 8)$, and the second one included maternal antibody titer $<1: 8$, 1:8 < 1:32 and $\geq 1: 32$. Then, we compared the geometric mean titers (GMTs), geometric mean antibody fold increases (GMIs) and seroconversion rates of poliovirus type-specific neutralizing antibodies after vaccination among participants with different maternal poliovirus antibody levels.
\end{abstract}

Results: The GMTs and GMIs of three types of poliovirus antibodies after vaccination in maternal antibody negative participants were significantly higher than those in maternal antibody positive participants. The seroconversion rates of type II and type III poliovirus antibodies in maternal antibody positive participants were significantly lower than those in maternal antibody negative participants. Among participants with maternal antibody titer $<1: 8,1: 8 \sim<1: 32$ and $\geq 1: 32$, the GMTs and GMls of three types of poliovirus antibodies after vaccination showed a tendency to decline with the increasing of maternal antibody levels. The seroconversion rates of three types of poliovirus antibodies in participants with maternal antibody titer $\geq 1: 32$ were significantly lower than those in participants with maternal antibody titer $<1: 8$ and 1:8 $\sim$ 1:32.

Conclusions: Maternal poliovirus antibodies interfered with the immune responses of infants to poliovirus vaccines, and a high level of maternal antibodies exhibited a greater dampening effect.

Trial registration: ClinicalTrials.gov NCT04264598 February 11, 2020; ClinicalTrials.gov NCT04264546 February 11, 2020; ClinicalTrials.gov NCT03902054 April 3, 2019. Retrospectively registered.

Keywords: Poliovirus vaccine, Maternal antibody, Immune response

\footnotetext{
* Correspondence: jscdclq@126.com

†'Siyue Jia and Rong Tang contributed equally to this work.

'Jiangsu Provincial Center for Disease Control and Prevention, NO. 172

Jiangsu Rd., Gulou District, Nanjing 210009, Jiangsu Province, China

Full list of author information is available at the end of the article
}

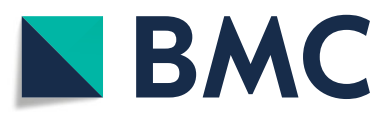

(c) The Author(s). 2020 Open Access This article is licensed under a Creative Commons Attribution 4.0 International License, which permits use, sharing, adaptation, distribution and reproduction in any medium or format, as long as you give appropriate credit to the original author(s) and the source, provide a link to the Creative Commons licence, and indicate if changes were made. The images or other third party material in this article are included in the article's Creative Commons licence, unless indicated otherwise in a credit line to the material. If material is not included in the article's Creative Commons licence and your intended use is not permitted by statutory regulation or exceeds the permitted use, you will need to obtain permission directly from the copyright holder. To view a copy of this licence, visit http://creativecommons.org/licenses/by/4.0/. The Creative Commons Public Domain Dedication waiver (http://creativecommons.org/publicdomain/zero/1.0/) applies to the data made available in this article, unless otherwise stated in a credit line to the data. 


\section{Background}

Polio was once a disease feared worldwide, which has been reduced by $99 \%$ due to the implementation of the Global Polio Eradication Initiative [1]. On October 17, 2019, World Health Organization (WHO) declared that wild poliovirus type II and type III have been eradicated worldwide [2]. While polio is a distant memory in most of the world, the disease still exists in some places and mainly affects children under five. Oral poliovirus vaccine (OPV) and inactivated poliovirus vaccine (IPV) are currently used to prevent polio across the world. OPV is one of the safest vaccines ever developed, which can be given to sick children and newborns [3]. On extremely rare occasions, the attenuated virus in OPV can mutate and regain virulence [4]. Some countries have switched from OPV to IPV to decrease the risk of emerging virulent poliovirus revertants. Since 2000, the United States has replaced OPV with IPV to eliminate the risk of vaccine-derived polio [5], while OPV is still used in some parts of the world, especially in developing countries, because of its cheapness. At present, there are two IPVs on the market, namely Sabin IPV and Salk IPV. The production and quality control of Salk IPV require at least a biosafety level 3 containment facility, while those of IPV based on Sabin strains will have a lower biosafety risk, and increase the availability and affordability of IPV in the low- or middle-income countries [6, 7].

Maternal antibodies are transferred to infants via the placenta during the third trimester of pregnancy and provide passive immunity to the newborns from infections during their first few months of life [8]. However, passively acquired maternal antibodies have been found to impair the immune responses of infants to measles [9], hepatitis A [10] and hepatitis B [11] vaccines. The results of a meta-analysis showed that maternal antibody concentrations and infant age at first vaccination both influence infant vaccine responses, and these effects are seen for almost all vaccines in the global immunization programs and influence the immune responses for some vaccines even at the age of 2 years [12]. Several studies have shown that maternal antibodies may impair the immune responses of infants to Salk IPV as well [13-16].

As WHO recommends Sabin IPV in the development of affordable next generation IPVs, and more countries look to move towards IPV schedules, it is critical to determine the effect of maternal antibodies on the response to Sabin IPV so as to optimize the effectiveness of it in infants. We have conducted three clinical trials to evaluate the safety and immunogenicity of an IPV based on Sabin strains. In this study, we pooled the data from these three clinical trials to investigate the effect of maternal poliovirus antibodies on the immune responses of infants to poliovirus vaccines, including the investigational Sabin IPV with different antigen contents, licensed Sabin IPV and Salk IPV.

\section{Methods}

Study design

Between August, 2017, and December, 2018, we conducted three clinical trials to evaluate the safety and immunogenicity of an IPV based on Sabin strains at two sites in Lianshui County and Dafeng District, Jiangsu Province, China. In the phase I trial (ClinicalTrials.gov: NCT04264598), 60 infants aged 2 months were randomly assigned in a 1:1:1 ratio to receive the mediumdose Sabin IPV, control Sabin IPV or control Salk IPV. In the phase Ib trial (ClinicalTrials.gov: NCT04264546), 20 infants aged 2 months were administered with the high-dose Sabin IPV, with no control group. In the phase II trial (ClinicalTrials.gov: NCT03902054), 600 infants aged 2 months were randomly assigned equally to receive the low-dose Sabin IPV, medium-dose Sabin IPV, high-dose Sabin IPV, control Sabin IPV or control Salk IPV. All infants were administered with 3 doses of IPV at 2, 3, and 4 months of age. Blood samples were collected immediately before the first dose and 30 days after the third dose for serum poliovirus type-specific neutralizing antibody detection, which was performed by the Chinese National Institute for Food and Drug Control, according to the method recommended by the WHO [17]. All Infants who completed the three-dose vaccination and had pre- and post-vaccination antibody detection results were included in the pooled analysis. According to the poliovirus neutralizing antibodies before vaccination, which could be considered as maternal antibodies, the infants in each group were reclassified. We used two classification methods. The first method was to divide the infants in each group into two subgroups, namely maternal antibody negative $(<1: 8)$ and maternal antibody positive $(\geq 1: 8)$. The second method was to divide the infants in each group into three subgroups, namely maternal antibody titer $<1: 8,1: 8 \sim<1: 32$ and $\geq 1: 32$. After that, we compared the geometric mean titers (GMTs), geometric mean antibody fold increases (GMIs) and seroconversion rates of poliovirus typespecific neutralizing antibodies after vaccination among participants with different maternal poliovirus antibody levels, to investigate the effect of maternal poliovirus antibodies on the immune responses of infants to poliovirus vaccines.

\section{Participants}

The population of Lianshui County is about 850,000, and that of Dafeng District is about 700,000. The total number of infants aged $0-12$ months at the two sites is about 18,000, which the trial participants were recruited from. The inclusion and exclusion criteria for infant participants in phase I, Ib and II trials were consistent. Eligible participants were healthy infants aged between 60 and 90 days, with no history and contraindication of 
poliovirus vaccination. Exclusion criteria were a history of polio, premature or low birth weight, congenital malformation or developmental disorders, a history of seizure or mental diseases, immunodeficiency or receiving immunosuppressive therapy, receipt of any blood products in the past 3 months, receipt of any live attenuated vaccines in the past 14 days, receipt of any subunit or inactivated vaccines in the past 7 days, receipt of any other research drugs, or other factors that were not suitable for clinical trials according to the judgment of researchers.

\section{Ethics statement}

The protocols and informed consent documents for the three clinical trials in the pooled analysis were approved by the Institutional Review Board of the Jiangsu Provincial Center of Disease Control and Prevention. Written informed consent was obtained for the guardians of all participants. The clinical trials were done in accordance with the Declaration of Helsinki and Good Clinical Practice guidelines.

\section{Vaccines}

The investigational Sabin IPV (low-dose, medium-dose and high-dose) were all developed by Beijing Minhai Biotechnology Co., Ltd. The control Sabin IPV and control Salk IPV were manufactured by Institute of Medical Biology Chinese Academy of Medical Sciences and Sanofi Pasteur S. A, respectively. The D antigen units (DU) of type I, type II and type III polioviruses in the investigational and control vaccines were as follows: lowdose Sabin IPV (10 DU, 30 DU and 30 DU), mediumdose Sabin IPV (15 DU, 45 DU and 45 DU), high-dose Sabin IPV (22 DU, 65 DU and 65 DU), control Sabin IPV (30 DU, 32 DU and 45 DU), control Salk IPV (40 DU, 8 DU and $32 \mathrm{DU})$. All vaccines were in liquid form, $0.5 \mathrm{ml}$ per dose.

\section{Statistical analysis}

Samples with antibody titers below the detection limit (1:8) were given an arbitrary value of 1:4 for calculations. Antibody titers were log-transformed in order to calculate the GMTs and GMIs. We used one-way ANOVA or Wilcoxon rank-sum test to compare the GMTs and GMIs of poliovirus type-specific neutralizing antibodies after vaccination among participants with different maternal poliovirus antibody levels. The seroconversion rates (defined as pre-vaccination titer less than 1:8 and post-vaccination titer $1: 8$ or more, or pre-vaccination titer 1:8 or more and at least four-fold increase postvaccination) among participants with different maternal poliovirus antibody levels were compared using $x^{2}$ test or Fisher's exact test. When a significant difference was found, further pairwise comparisons were performed and Bonferoni-adjusted $P$ values were calculated. All reported $P$ values were 2 sided, and values less than 0.05 were regarded as statistically significant. Statistical analyses were performed by using SAS 9.3 software (SAS Institute, Inc., Cary, NC, USA).

\section{Results \\ Study participants}

Figure 1 shows the pooled analysis profile. Six hundred nine infants who completed three-dose vaccination and blood collection were included in the pooled analysis. Of them, 109 received low-dose Sabin IPV, 125 received medium-dose Sabin IPV, 127 received high-dose Sabin IPV, 124 received control Sabin IPV, and 124 received control Salk IPV. Baseline demographic characteristics of the participants were similar among the five vaccine groups (Table 1). Table 2 shows the maternal poliovirus type-specific antibody levels of the participants. The positive rates of maternal antibodies in low-dose Sabin IPV group, medium-dose Sabin IPV group, high-dose Sabin IPV group, control Sabin IPV group and control Salk IPV group ranged from 70.87 to $78.40 \%$ for type I poliovirus, 34.40 to $44.95 \%$ for type II poliovirus, 16.54 to $26.61 \%$ for type III poliovirus. The GMTs of maternal antibodies in the five vaccine groups ranged from 17.57 to 21.21 for type I poliovirus, 6.44 to 8.39 for type II poliovirus, 5.33 to 6.41 for type III poliovirus. The majority of participants in the five vaccine groups had a maternal antibody titer of 1:8 < 1:32 or $\geq 1: 32$ for type I poliovirus, $<1: 8$ or $1: 8 \sim<1: 32$ for type II and type III polioviruses.

\section{Maternal antibody dichotomy}

Table 3 shows the comparisons of GMTs, GMIs and seroconversion rates of poliovirus antibodies after vaccination between maternal antibody negative and positive participants. The GMTs of antibodies after vaccination in maternal antibody negative participants were significantly higher than those in maternal antibody positive participants for type I poliovirus in low-dose Sabin IPV group $(P=0.006)$, high-dose Sabin IPV group $(P=0.025)$ and control Sabin IPV group $(P=0.001)$, for type II poliovirus in low-dose Sabin IPV group $(P=$ $0.002)$, medium-dose Sabin IPV group $(P=0.005)$, highdose Sabin IPV group $(P=0.008)$ and control Salk IPV group $(P<0.001)$, for type III poliovirus in low-dose Sabin IPV group $(P=0.017)$, medium-dose Sabin IPV group $(P=0.003)$ and control Sabin IPV group $(P<$ 0.001 ). The GMIs of three types of poliovirus antibodies in maternal antibody negative participants were significantly higher than those in maternal antibody positive participants in all five vaccine groups $(P<0.001)$. The seroconversion rates of maternal antibody negative participants for three types of polioviruses in the five 


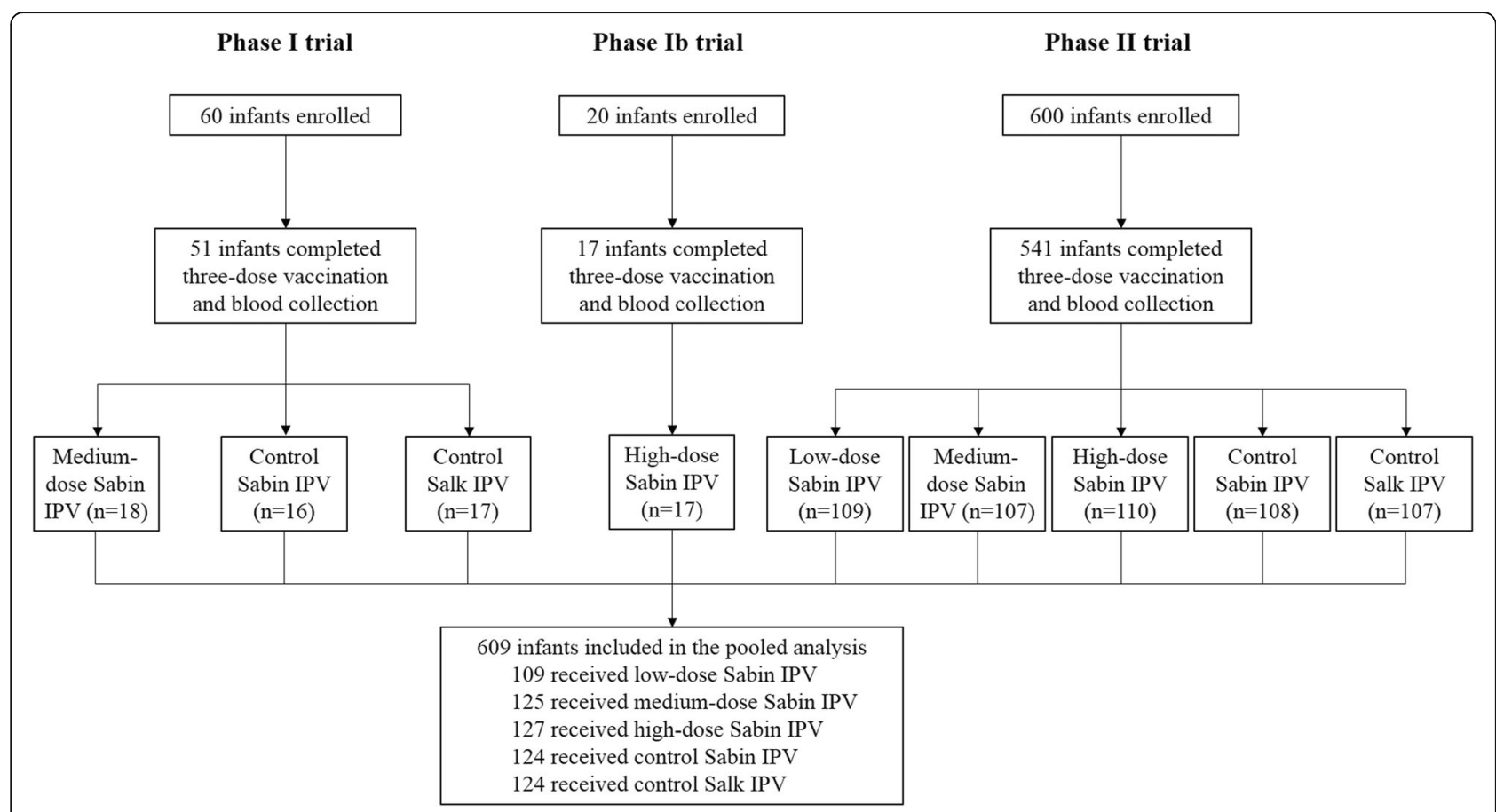

Fig. 1 Pooled analysis profile

vaccine groups were all $100 \%$. The seroconversion rates of type I poliovirus antibodies in all five vaccine groups were comparable between maternal antibody positive and negative participants $(P>0.05)$. The seroconversion rates of antibodies in maternal antibody positive participants were significantly lower than those in maternal antibody negative participants for type II poliovirus in low-dose Sabin IPV group $(P=0.008)$, medium-dose Sabin IPV group $(P=0.023)$, control Sabin IPV group $(P=0.014)$ and control Salk IPV group $(P<0.001)$, for type III poliovirus in low-dose Sabin IPV group $(P=0.001)$, medium-dose Sabin IPV group $(P=0.006)$, high-dose Sabin IPV group $(P=$ $0.004)$ and control Sabin IPV group $(P=0.001)$.

\section{Maternal antibody trichotomy}

Figure 2 shows the multiple comparisons of GMTs, GMIs and seroconversion rates of poliovirus type- specific neutralizing antibodies after vaccination among participants with different maternal poliovirus antibody levels. Among participants with maternal antibody titer $<1: 8,1: 8 \sim<1: 32$ and $\geq 1: 32$, the GMTs and GMIs of poliovirus antibodies after vaccination showed a tendency to decline with the increasing of maternal antibody levels. Significant differences in the GMTs after vaccination were found among participants with maternal antibody titer $<1: 8,1: 8 \sim<1: 32$ and $\geq 1: 32$ for type I poliovirus in low-dose Sabin IPV group, medium-dose Sabin IPV group, high-dose Sabin IPV group and control Sabin IPV group, for type II poliovirus in all five vaccine groups, for type III poliovirus in low-dose Sabin IPV group, medium-dose Sabin IPV group and control Sabin IPV group. Besides, the GMIs of three types of poliovirus antibodies in all five vaccine groups were significantly different among participants with different maternal poliovirus antibody levels. The seroconversion

Table 1 Baseline characteristics of the study participants

\begin{tabular}{|c|c|c|c|c|c|}
\hline Characteristics & Group 1 & Group 2 & Group 3 & Group 4 & Group 5 \\
\hline $\mathrm{N}$ & 109 & 125 & 127 & 124 & 124 \\
\hline Age-days ${ }^{a}$ & $72.18 \pm 8.93$ & $73.00 \pm 8.81$ & $72.59 \pm 8.81$ & $72.12 \pm 8.09$ & $73.83 \pm 7.89$ \\
\hline \multicolumn{6}{|l|}{ Sex-no. (\%) } \\
\hline Male & $49(44.95)$ & $68(54.40)$ & 65 (51.18) & 61 (49.19) & $63(50.81)$ \\
\hline Female & $60(55.05)$ & 57 (45.60) & 62 (48.82) & $63(50.81)$ & 61 (49.19) \\
\hline
\end{tabular}

Group 1: low-dose Sabin IPV group. Group 2: medium-dose Sabin IPV group. Group 3: high-dose Sabin IPV group. Group 4: control Sabin IPV group. Group 5: control Salk IPV group

aPlus-minus values are means \pm SD 
Table 2 Maternal poliovirus type-specific antibody levels of the study participants

\begin{tabular}{|c|c|c|c|c|c|c|c|c|}
\hline \multirow[t]{2}{*}{ Group } & \multirow[t]{2}{*}{$\mathrm{N}$} & \multirow[t]{2}{*}{ Type } & \multirow{2}{*}{$\begin{array}{l}\text { Positive } \\
\text { participants }\end{array}$} & \multirow{2}{*}{$\begin{array}{l}\text { Positive } \\
\text { rate (\%) }\end{array}$} & \multirow[t]{2}{*}{ GMT } & \multicolumn{3}{|c|}{ Participants with different titers (1:) } \\
\hline & & & & & & $<8$ & $8 \sim<32$ & $\geq 32$ \\
\hline \multirow[t]{3}{*}{1} & \multirow[t]{3}{*}{109} & । & 82 & 75.23 & 17.57 & 27 & 47 & 35 \\
\hline & & $\|$ & 49 & 44.95 & 8.39 & 60 & 33 & 16 \\
\hline & & III & 29 & 26.61 & 6.13 & 80 & 20 & 9 \\
\hline \multirow[t]{3}{*}{2} & \multirow[t]{3}{*}{125} & । & 98 & 78.40 & 18.44 & 27 & 50 & 48 \\
\hline & & $\|$ & 43 & 34.40 & 6.73 & 82 & 30 & 13 \\
\hline & & III & 23 & 18.40 & 5.33 & 102 & 19 & 4 \\
\hline \multirow[t]{3}{*}{3} & \multirow[t]{3}{*}{127} & । & 90 & 70.87 & 17.99 & 37 & 39 & 51 \\
\hline & & $\|$ & 46 & 36.22 & 6.69 & 81 & 35 & 11 \\
\hline & & III & 21 & 16.54 & 5.59 & 106 & 14 & 7 \\
\hline \multirow[t]{3}{*}{4} & \multirow[t]{3}{*}{124} & 1 & 96 & 77.42 & 21.21 & 28 & 40 & 56 \\
\hline & & II & 47 & 37.90 & 6.44 & 77 & 42 & 5 \\
\hline & & III & 33 & 26.61 & 6.41 & 91 & 23 & 10 \\
\hline \multirow[t]{3}{*}{5} & \multirow[t]{3}{*}{124} & 1 & 95 & 76.61 & 19.15 & 29 & 45 & 50 \\
\hline & & $\|$ & 46 & 37.10 & 6.92 & 78 & 36 & 10 \\
\hline & & III & 31 & 25.00 & 5.79 & 93 & 25 & 6 \\
\hline
\end{tabular}

Group 1: low-dose Sabin IPV group. Group 2: medium-dose Sabin IPV group. Group 3: high-dose Sabin IPV group. Group 4: control Sabin IPV group. Group 5: control Salk IPV group

rates of three types of poliovirus antibodies in all five groups were comparable between participants with maternal antibody titer $<1: 8$ and 1:8 $<1: 32$, ranged from 94.44 to $100 \%$. While the seroconversion rates of antibodies in participants with maternal antibody titer $\geq 1: 32$ were significantly lower for type I poliovirus in control Salk IPV group, for type II poliovirus in all five vaccine groups, for type III poliovirus in low-dose Sabin IPV group, medium-dose Sabin IPV group, high-dose Sabin IPV group and control Sabin IPV group, ranged from 25.00 to $81.81 \%$.

\section{Discussion}

In our study, a certain weakening effect of maternal poliovirus antibodies on the immune responses of infants to poliovirus vaccines was observed, including the investigational Sabin IPV with different antigen contents, licensed Sabin IPV and Salk IPV. Among the five vaccine groups, the seropositive rates of maternal poliovirus type-specific antibodies were the highest for type I poliovirus, ranged from 70.87 to $78.40 \%$, followed by those for type II poliovirus, ranged from 34.40 to $44.95 \%$, and those for type III poliovirus were the lowest, ranged from 16.54 to $26.61 \%$. The seropositive rates of maternal poliovirus type-specific antibodies in this study were similar to the results of a recent phase III IPV clinical trial [18]. This is because after years of OPV vaccination in China, high levels of poliovirus antibodies have formed in the population, resulting in high levels of maternal antibodies in newborns.
In the investigational Sabin IPV, control Sabin IPV and control Salk IPV groups, the effect of maternal poliovirus antibodies on poliovirus vaccines were consistent, this suggested that this effect may be a common problem in IPV. The results of maternal antibodies lowered the antibody responses of infants to IPV were also found in other randomized clinical trials [13-16], and this finding of our study is in accordance with that of a meta-analysis, where 2-fold higher maternal antibody concentrations resulted in 20 to $28 \%$ lower post-vaccination antibody concentration of IPV [12]. In another similar study conducted by us, the immune responses of IPV were attenuated by the high level of maternal poliovirus antibodies, but an opposite result showed that the post-vaccination GMTs for type I poliovirus were significantly higher among infants with high maternal antibody levels in Salk IPV group [19]. The exceptional result seemed to lack biological rationality, or it might be due to the small sample size and insufficient sample representativeness. Moreover, the negative effect of maternal poliovirus antibodies on the immune responses of poliovirus vaccines were found in low-dose Sabin IPV group, medium-dose Sabin IPV group and highdose Sabin IPV group, which indicated that the increased antigen contents of polioviruses in vaccines may not diminish this effect of maternal poliovirus antibodies.

There were several limitations of this study. First, the blood samples for maternal poliovirus antibody detection were collected immediately before the first dose, when the infant participants were 2 months old, not the cord blood. But China has been certified as being polio free since 2000 [20], it is extremely unlikely for an infant 


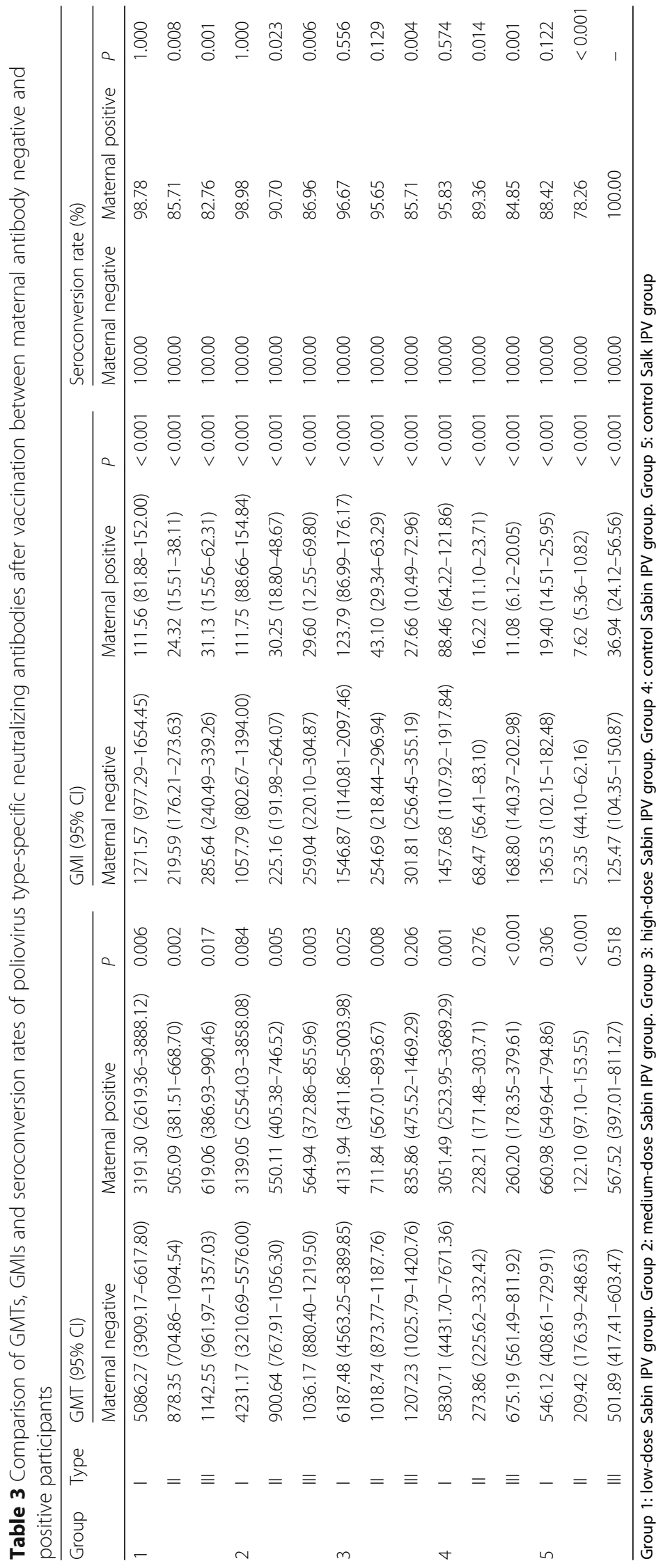




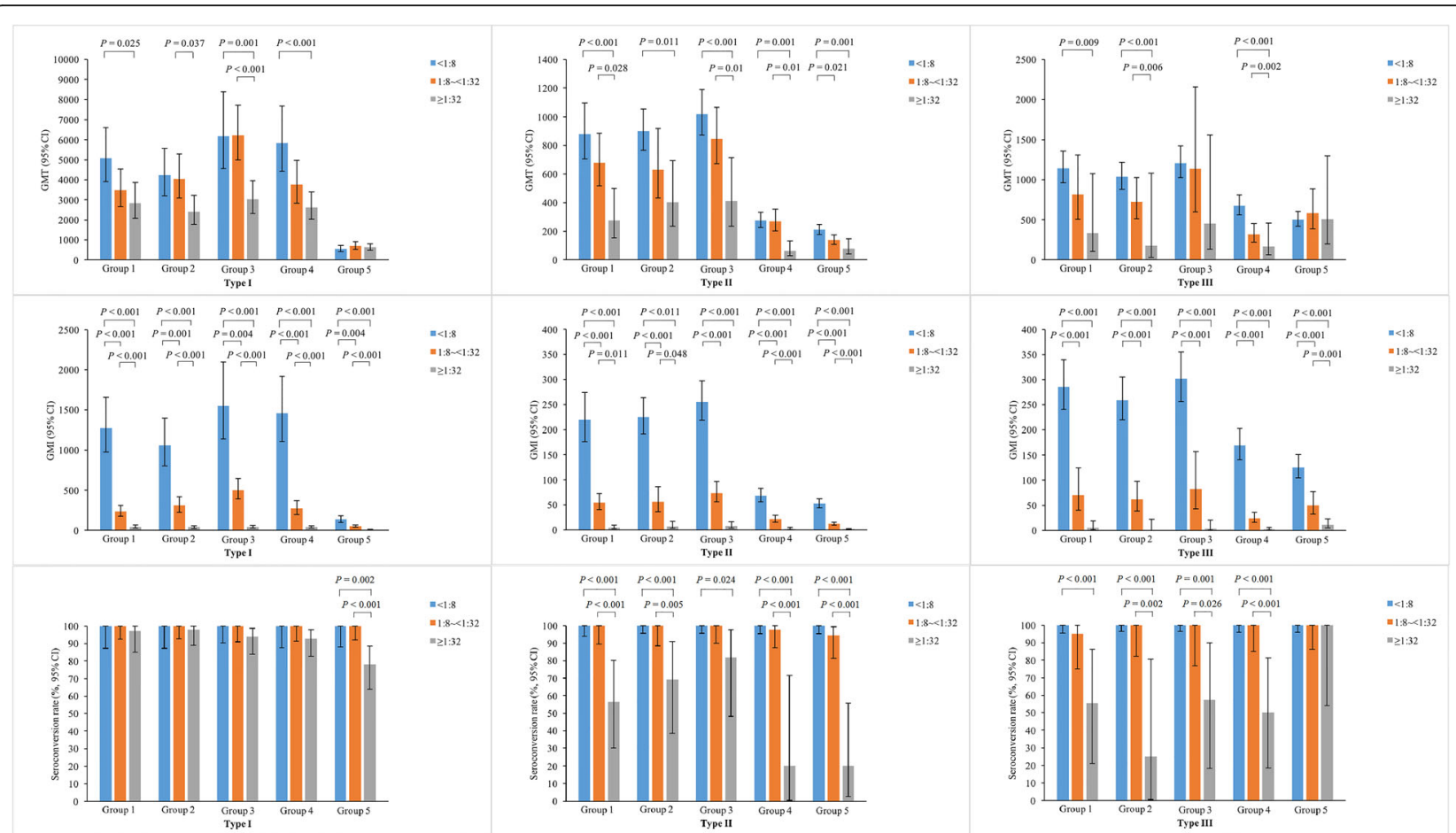

Fig. 2 Multiple comparisons of GMTs, GMIs and seroconversion rates of poliovirus type-specific neutralizing antibodies after vaccination among participants with different maternal poliovirus antibody levels. Group 1: low-dose Sabin IPV group. Group 2: medium-dose Sabin IPV group. Group 3: high-dose Sabin IPV group. Group 4: control Sabin IPV group. Group 5: control Salk IPV group

to be infected with poliovirus within 2 months of birth. Thus, it is reasonable to consider pre-vaccination poliovirus antibodies as the maternal antibodies. Second, for type II and type III polioviruses, the proportion of participants with maternal antibody titer $\geq 1: 32$ was relatively small. This may have some influence on the results, and needs further study. Third, this study is a pooled analysis of three IPV clinical trials, not a randomized controlled study designed specifically to explore the effect of maternal poliovirus antibodies on the immune responses of infants to poliovirus vaccines.

Although the seroconversion rates of infants with high maternal antibodies are lower, the GMTs of them after immunization are still relatively high, which may protect them against polio. In addition, the presence of maternal antibodies can also provide protection for infants during their first few months of life. Considering the relatively poor immunogenicity of IPV in infants who were maternal antibody positive, especially in those with maternal antibody titer $\geq 1: 32$, an adjusted immunization strategy of IPV may be needed for them, such as delaying the vaccination. Besides, the effect of maternal poliovirus antibodies on the antibody persistence of IPV vaccination may be needed to be observed in long term, and we will take this as a potential focus of our subsequent work. In the future, maternal antibody will be an important factor to be considered in achieving precision immunization in infants [21].

\section{Conclusions}

Overall, maternal poliovirus antibodies interfered with the immune responses of infants to poliovirus vaccines, and a high level of maternal antibodies exhibited a greater dampening effect.

\section{Abbreviations}

OPV: Oral poliovirus vaccine; IPV: Inactivated poliovirus vaccine;

GMTs: Geometric mean titers; GMIs: Geometric mean antibody fold increases

\section{Acknowledgments}

We thank all participants who contributed to this work.

\section{Authors' contributions}

QL designed the study and contributed to the revision of the manuscript. SJ and RT contributed to drafting of the manuscript and data interpretation. GL and $\mathrm{YH}$ contributed to data collection, data management and statistical analysis. Each author listed on the manuscript has seen and approved the submission of this version of the manuscript.

\section{Funding}

No funding of any kind has been received for this study.

\section{Availability of data and materials}

All authors had full access to the data and materials. Data are available within this article. Detailed data is available from the corresponding author upon reasonable request. 


\section{Ethics approval and consent to participate}

The study was approved by the Medical Ethics Committee of the Jiangsu Provincial Center for Disease Control and Prevention (Approval Number: JSJK2017-A006-02; JSJK2017-A013-02). Written informed consent was obtained for the guardians of all participants.

\section{Consent for publication}

Not applicable since there are no details, images, or videos relating to an individual person.

\section{Competing interests}

Guifan Li is an employee of Beijing Minhai Biotechnology Co., Ltd. The authors have no other relevant affiliations or financial involvement with any organization or entity with a financial interest in or financial conflict with the subject matter or materials discussed in the manuscript apart from those disclosed.

\section{Author details}

'Jiangsu Provincial Center for Disease Control and Prevention, NO. 172 Jiangsu Rd., Gulou District, Nanjing 210009, Jiangsu Province, China. ${ }^{2}$ Beijing Minhai Biotechnology Co. Ltd., Beijing, China.

Received: 18 February 2020 Accepted: 16 August 2020

Published online: 31 August 2020

\section{References}

1. World Health Organization. Polio eradication. https://www.who.int/newsroom/facts-in-pictures/detail/polio-eradication. Accessed 10 Jan 2020.

2. World Health Organization. Two out of three wild poliovirus strains eradicated. https://www.who.int/news-room/feature-stories/detail/two-outof-three-wild-poliovirus-strains-eradicated. Accessed 15 Jan 2020.

3. World Health Organization. Global Polio Eradication Initiative. http:// polioeradication.org/polio-today/faq/. Accessed 2 Feb 2020.

4. Heinsbroek E, Ruitenberg EJ. The global introduction of inactivated polio vaccine can circumvent the oral polio vaccine paradox. Vaccine. 2010;28(22): 3778-83.

5. Centers for Disease Control and Prevention. What are the Types of Polio Vaccine? https://www.cdc.gov/vaccines/vpd/polio/public/. Accessed 10 Feb 2020.

6. Liao G, Li R, Li C, Sun M, Li Y, Chu J, Jiang S, Li Q. Safety and immunogenicity of inactivated poliovirus vaccine made from Sabin strains: a phase II, randomized, positive-controlled trial. J Infect Dis. 2012;205(2):23743.

7. Verdijk P, Rots NY, van Oijen MGCT, Oberste MS, Boog CJ, Okayasu H, Sutter RW, Bakker WAM. Safety and immunogenicity of inactivated poliovirus vaccine based on Sabin strains with and without aluminum hydroxide: a phase I trial in healthy adults. Vaccine. 2013;31(47):5531-6.

8. Vojtek I, Dieussaert I, Doherty TM, Franck V, Hanssens L, Miller J, BekkatBerkani R, Kandeil W, Prado-Cohrs D, Vyse A. Maternal immunization: where are we now and how to move forward? Ann Med. 2018;50(3):193-208.

9. Albrecht $P$, Ennis FA, Saltzman EJ, Krugman S. Persistence of maternal antibody in infants beyond 12 months: mechanism of measles vaccine failure. J Pediatr. 1977;91(5):715-8.

10. Letson GW, Shapiro CN, Kuehn D, Gardea C, Welty TK, Krause DS, Lambert $\mathrm{SB}$, Margolis HS. Effect of maternal antibody on immunogenicity of hepatitis A vaccine in infants. J Pediatr. 2004;144(3):327-32.

11. Hu Y, Wu Q, Xu B, Zhou Z, Wang Z, Zhou Y-H. Influence of maternal antibody against hepatitis $B$ surface antigen on active immune response to hepatitis B vaccine in infants. Vaccine. 2008;26(48):6064-7.

12. Voysey M, Kelly DF, Fanshawe TR, Sadarangani M, O'Brien KL, Perera R, Pollard AJ. The influence of maternally derived antibody and infant age at vaccination on infant vaccine responses: an individual participant metaanalysis. JAMA Pediatr. 2017;171(7):637-46.

13. Asturias E, Dueger E, Omer S, Melville A, Nates $S$, Laassri M, Chumakov K, Halsey N. Randomized trial of inactivated and live polio vaccine schedules in Guatemalan infants. J Infect Dis. 2007;196(5):692-8.

14. Dayan G, Thorley M, Yamamura Y, Rodríguez N, McLaughlin S, Torres L, Seda A, Carbia M, Alexander L, Caceres V. Serologic response to inactivated poliovirus vaccine: a randomized clinical trial comparing 2 vaccination schedules in Puerto Rico. J Infect Dis. 2007;195(1):12-20.
15. Sormunen H, Stenvik M, Eskola J, Hovi T. Age- and dose-interval-dependent antibody responses to inactivated poliovirus vaccine. J Med Virol. 2001;63(4): $305-10$.

16. Linder N, Handsher R, German B, Sirota L, Bachman M, Zinger S, Mendelson E, Barzilai A. Controlled trial of immune response of preterm infants to recombinant hepatitis $B$ and inactivated poliovirus vaccines administered simultaneously shortly after birth. Arch Dis Child Fetal Neonatal Ed. 2000; 83(1):F24-7.

17. World Health Organization. Manual for the virological investigation of polio. http://www.who.int/mediacentre/factsheets/fs114/en/. Accessed 24 Nov 2011.

18. Hu Y, Xu K, Han W, Chu K, Jiang D, Wang J, Tian X, Ying Z, Zhang Y, Li C, et al. Safety and Immunogenicity of Sabin Strain Inactivated Poliovirus Vaccine Compared With Salk Strain Inactivated Poliovirus Vaccine, in Different Sequential Schedules With Bivalent Oral Poliovirus Vaccine: Randomized Controlled Noninferiority Clinical Trials in China. Open Forum Infect Dis. 2019;6(10):ofz380.

19. Tang R, Chu K, Hu Y, Chen L, Zhang M, Liu S, Ma H, Wang J, Zhu F, Hu Y, et al. Effect of maternal antibody on the infant immune response to inactivated poliovirus vaccines made from Sabin strains. Hum Vaccin Immunother. 2019;15(5):1160-6.

20. Adams A, Boualam L, Diorditsa S, Gregory C, Jee Y, Mendoza-Aldana J, Roesel S. Maintaining polio-free certification in the World Health Organization Western Pacific region for over a decade. J Infect Dis. 2014; 210(suppl 1):S259-67.

21. Jia S, Li J, Liu Y, Zhu F. Precision immunization: a new trend in human vaccination. Hum Vaccin Immunother. 2020:1-10.

\section{Publisher's Note}

Springer Nature remains neutral with regard to jurisdictional claims in published maps and institutional affiliations.
Ready to submit your research? Choose BMC and benefit from:

- fast, convenient online submission

- thorough peer review by experienced researchers in your field

- rapid publication on acceptance

- support for research data, including large and complex data types

- gold Open Access which fosters wider collaboration and increased citations

- maximum visibility for your research: over $100 \mathrm{M}$ website views per year

At BMC, research is always in progress.

Learn more biomedcentral.com/submissions 